\title{
Interpretation of Line-Integrated Signals from 2-D Phase Contrast Imaging on LHD
}

\author{
Clive MICHAEL, Kenji TANAKA, Leonid VYACHESLAVOV ${ }^{1)}$, Andrei SANIN ${ }^{1)}$, \\ Kazuo KAWAHATA and S. OKAJIMA \\ National Institute of Fusion Science, Toki 509-0293, Japan \\ 1) Budker Institute for Nuclear Physics, Novisibirsk, Russia
}

(Received 8 December 2006 / Accepted 22 May 2007)

\begin{abstract}
Two dimensional (2D) phase contrast imaging (PCI) is an excellent method to measure core and edge turbulence with good spatial resolution $(\Delta \rho \sim 0.1)$. General analytical consideration is given to the signal interpretation of the line-integrated signals, with specific application to images from 2D PCI. It is shown that the Fourier components of fluctuations having any non-zero component propagating along the line of sight are not detected. The ramifications of this constraint are discussed, including consideration of the angle between the sight line and flux surface normal. In the experimental geometry, at the point where the flux surfaces are tangent to the sight line, it is shown that it may be possible to detect large poloidally extended (though with small radial wavelength) structures, such as GAMS. The spatial localization technique of this diagnostic is illustrated with experimental data.

(c) 2007 The Japan Society of Plasma Science and Nuclear Fusion Research
\end{abstract}

Keywords: plasma,imaging,maximum entropy, correlation, turbulence, phase contrast imaging, line-integration

DOI: $10.1585 /$ prr.2.S1034

\section{Inroduction}

Phase contrast imaging (PCI) is a well established technique $[1,2]$ to image density fluctuations, integrated along a line of sight. The technique enables good wavenumber resolution and the propagation direction and velocity perpendicular to the line of sight can be analyzed. The system installed on LHD [2-5] is the first of its type to employ a 2D imaging principle, rather than conventional 1D imaging, which gives the additional capability to spatially resolve fluctuations along the line of sight. Other such systems employing 1-D imaging are reported in [6,7].

The purpose of this paper is to present a novel analysis of the interpretation of line-integrated signals and to understand what these results imply for the detection of radial and poloidal wave-vector components in flux coordinates. These results are applicable to any type of fluctuation signal, not just from PCI.

The phase contrast imaging system employs a $\mathrm{CO}_{2}$ laser at $10.6 \mu \mathrm{m}$. The scattering angle of plasma fluctuations of $\mathrm{mm}$ scale is small. The scattering is in the "RamanNath" regime, sothat the registered image is a line-integral. The optical system employs a quarter wave groove at the primary beam focus to convert phase to amplitude. The size of the phase plate and expanded beam size defines a lower limit to the measurable $k$.

For the geometry consider a coordinate axis such that the $z$ axis is directed along the line of sight (probing laser beam). Spatial localization along the line of sight can be

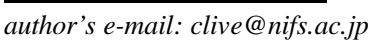

obtained by considering the two constraints: (1) That the toroidal wavelength is considerably larger than perpendicular to $B$, so that:

$$
\boldsymbol{k}(\boldsymbol{r}) \cdot \boldsymbol{B}(\boldsymbol{r})=0
$$

(where $\boldsymbol{r}=(x, y, z)$ is the position vector and $\boldsymbol{k}$ is wavevector conjugate to the spatial coordinate $\boldsymbol{r}+\boldsymbol{\delta} \boldsymbol{r}$, where $\boldsymbol{\delta} \boldsymbol{r}$ is a small displacement about $\boldsymbol{r}$; the conceptual meaning of this separation is discussed in Sec. 2), and (2) that PCI detected signal is insensitive to any components with $k_{z} \neq 0$, because of line-integration effects (discussed in Sec. 1). From these two facts, the measured feature with wave-number components $\left(k_{x}, k_{y}\right)$ in the $2 \mathrm{~d}$ image plane (for which $k_{z}=0$ ) must be perpendicular to the projection of the associated local magnetic field vector in the image plane, $\left(B_{x}, B_{y}\right)$, since $k_{x} B_{x}+k_{y} B_{y}=0$. In this way, from the spatial power spectrum of a $2 \mathrm{D}$ image of the fluctuations, $\bar{S}\left(k_{x}, k_{y}\right)$, the wave-number resolved fluctuation profile can be determined given that the magnetic field direction,

$$
\chi_{p}(z)=\tan ^{-1}\left(B_{y}(z) / B_{x}(z)\right),
$$

is a unique function of $z$. On LHD, a system has been developed with a $6 \times 82 \mathrm{D}$ detector to take advantage of this technique [3]. The pitch angle variation along the line of sight is around $\sim 80^{\circ}$, so good separation of fluctuation components is possible. The system images fluctuations within the diameter of the probing laser beam having wave-numbers in the range $0.1<k<0.6 \mathrm{~mm}^{-1}$, or by changing the magnification, up to $k<4 \mathrm{~mm}^{-1}[2,5]$. The 


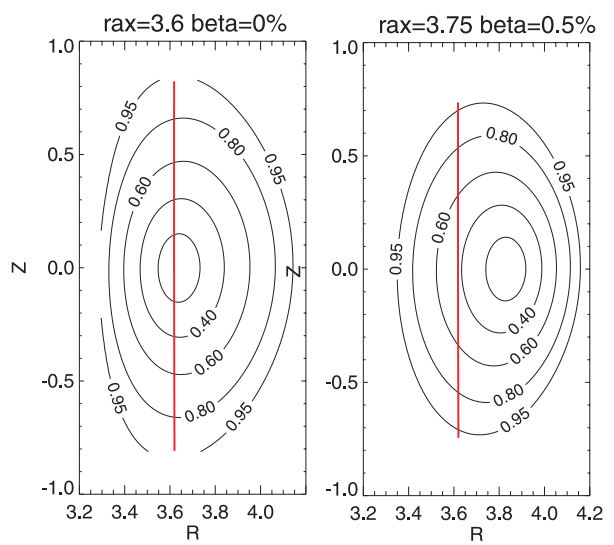

Fig. 1 Sight line geometry in different magnetic configurations.

sight line of the system relative to the magnetic surfaces on LHD, for two typical magnetic configurations is indicated in Fig. 1 (however the thickness of the probing laser beam, with $1 / e^{2}$ diameter $\sim 10 \mathrm{~cm}$, is not represented). The sightline penetrates from the edge to the core, depending on the magnetic axis position of the plasma $\left(R_{\mathrm{ax}}\right)$, implying that the entire fluctuation amplitude profile $\tilde{n}(\rho), 0<\rho<1$ can be obtained.

The structure of this paper is organized as follows. In Sec. 1, we demonstrate the localization principle with respect to experimental measurements. In Sec. 2, we describe the general interpretation of line-integrated fluctuation signals. We present a different formalism than what has been presented before $[8,9]$, though the results are fundamentally the same. In Sec. 3, we consider which fluxcoordinate resolved Fourier components (poloidal and/or radial) appear in the line-integrated signal.

\section{Image Processing of 2D PCI Images}

The raw image sequence $\tilde{N}(x, y, t)$ is first preprocessed to generate a correlation image $\Gamma(\Delta x, \Delta y, f)$, where $f$ is the temporal frequency and $\Delta x, \Delta y$ is the spatial separation in the image. An image of such a fluctuation is plotted in Fig. 2 (a). It is clear that there are structures oriented at both $\sim \pm 40^{\circ}$ corresponding to fluctuations from the top and bottom of the sight line. The spatial power spectrum $S\left(k_{x}, k_{y}\right)$ is then computed by applying the Fourier transform, however, generally, to improve spatial resolution, we use high resolution techniques such as Maximum Entropy to reduce the broadening. Finally, by transforming from rectangular to polar coordinates $k_{p}=\sqrt{k_{x}^{2}+k_{y}^{2}}, \chi=\tan ^{-1}\left(k_{y} / k_{x}\right)+\pi / 2$, the fluctuation is localized to $\rho$ according to a known field line dispersion $\chi_{p}(\rho)$, as is shown in Fig. 2 (b). The reversal of the direction of the $k$ peaks near the top and bottom is a result of the existence of a unique poloidal propagation direction, in this case being in the electron diamagnetic direction, as explained in Fig. 4 (b). Further consideration of this is given in Sec. 3 .
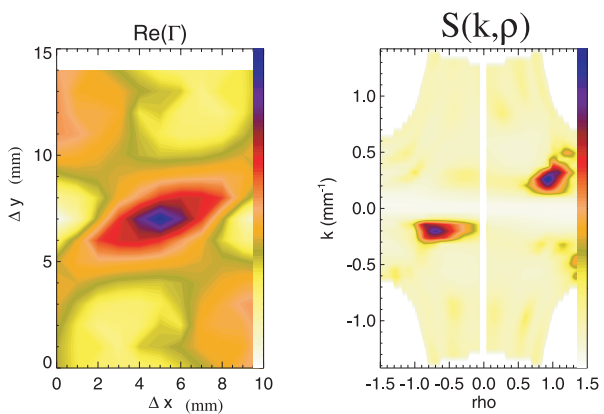

Fig. 2 (a) Correlation image. (b) Spatial and k distribution of fluctuation power.

\section{Line-Integral of Turbulence Spec- trum}

Define the local density fluctuation $\tilde{n}(x, y, z)$ and line integrated density fluctuation $\tilde{N}(x, y)=\int_{L} \tilde{n}(x, y, z) d z$ where the $\mathrm{z}$ axis is along the sight line of the probing beam denoted by $L$. We define local and line-integrated correlations as $\Gamma(\Delta x, \Delta y, \Delta z ; x ; y ; z)=\langle(\tilde{n}(x, y, z) \tilde{n}(x+\Delta x, y+$ $\Delta y, z+\Delta z)\rangle$ and $\bar{\Gamma}(\Delta x, \Delta y ; x ; y)=\langle\tilde{N}(x, y) \tilde{N}(x+\Delta x, y+\Delta y)$. Angle brackets denote ensemble averaging, either in the time or frequency domain. Though strictly the time or frequency dependance should be treated formally, this is not treated here because it does not affect the line integral effects. Spatial homogeneity transverse to the beam is assumed (since the beam size is small with respect to the plasma size) so the $x$ and $y$ dependencies can be dropped. From these definitions, it can be shown [8] that $\bar{\Gamma}$ is related to $\Gamma$ through:

$$
\bar{\Gamma}(\Delta x, \Delta y)=\int_{L} \int_{L} \Gamma(\Delta x, \Delta y, \Delta z ; z) d \Delta z d z
$$

We define the spectral density functions $\bar{S}\left(k_{x}, k_{y}\right)$ as the 2D Fourier transform of the line integrated correlation function $\bar{\Gamma}(\Delta x, \Delta y)$, and $S\left(k_{x}, k_{y}, k_{z} ; z\right)$ as the 3D Fourier transform of $\Gamma(\Delta x, \Delta y, \Delta z ; z)$ for fixed $z$. By combining these definitions with the Fourier transform of Eq. (3) over $\Delta x, \Delta y$, and substituting the RHS for a single inverse Fourier transform of $S\left(k_{x}, k_{y}, k_{z} ; z\right)$ over $k_{z}$, the following equation can be written:

$$
\begin{aligned}
\bar{S}\left(k_{x}, k_{y}\right)= & (2 \pi)^{-1} \int_{-\infty}^{\infty} \int_{-\infty}^{\infty} \int_{L} S\left(k_{x}, k_{y}, k_{z} ; z\right) \\
& \times \exp \left(-i k_{z} \Delta z\right) d k_{z} d \Delta z d z .
\end{aligned}
$$

By swapping the order of integration of $d \Delta z$ and $d k_{z}$, the term $\int_{-\infty}^{\infty} \exp \left(-i k_{z} \Delta z\right) d \Delta z$ is replaced to $\delta\left(k_{z}\right)$, and the final result is:

$$
\bar{S}\left(k_{x}, k_{y}\right)=(2 \pi)^{-1} \int S\left(k_{x}, k_{y}, 0 ; z\right) d z .
$$

Thus, the line integrated power spectrum is only sensitive to local fluctuation spectral components with $k_{z}=0$. In other words, all other components cancel out. The above 
equation is dimensionally correct, since $S$ being local power spectral density has dimensions of $\mathrm{m}^{-3}$ and $\bar{S}$ has dimensions of $\mathrm{m}^{-2}$. The above equation is analogous to the projection slice theorem in Fourier theory/tomography. In order to make use of the Wiener-Kinchine theorem to relate the local power spectrum to the local correlation function, it is necessary to make the quasi-homogeneous approximation [10], that is $\Gamma(\Delta x, \Delta y, \Delta z ; z)=\Gamma\left(\Delta x, \Delta y, \Delta z ; z^{\prime}\right)$ for $\left|z^{\prime}-z\right|<\mathcal{L}$, where $\mathcal{L}$ is the correlation length. This is equivalent to the statement that the scale length for changes in the fluctuation properties $\left(l_{\text {turb }}=\left(d\left\langle\tilde{n}^{2}\right\rangle / d z\right)^{-1}\left\langle\tilde{n}^{2}\right\rangle\right)$ is larger than the correlation length, i.e. $l_{\text {turb }} \gg \mathcal{L}$.

The maximum desired spatial resolution $l_{\text {turb }}$ is therefore at least several times $\mathcal{L}$, or equivalently, $\lambda$, the fluctuation wavelength. However, as has been shown [5], the instrumental resolution is $l_{\text {instr }} \sim a \lambda / B_{w}$ where $B_{w}$ is the least of the width of the image/probing laser beam and $a$ is the plasma minor radius (however this can be improved using high resolution spectral analysis, effectively increasing $B_{w}$ ). This is larger than the fluctuation wavelength by a factor $a / B_{w} \approx 10$ (for overview mode, sensitive to largest scale fluctuations).

This result in Eq. (5) can be related to the conventional definitions as follows. The rms local density fluctuation is given by $\left\langle\tilde{n}^{2}(z)\right\rangle=\int S\left(k_{x}, k_{y}, k_{z} ; z\right) d k_{x} d k_{y} d k_{z}$, and the line integrated density fluctuation has rms given by $\left\langle\tilde{N}^{2}\right\rangle=\int \bar{S}\left(k_{x}, k_{y}\right) d k_{x} d k_{y}$. Therefore, combining these definitions with Eq. (5), it is clear that

$$
\left\langle\tilde{N}^{2}\right\rangle=\int\left[\frac{\iint S\left(k_{x}, k_{y}, 0 ; z\right) d k_{x} d k_{y}}{\left\langle\tilde{n}^{2}(z)\right\rangle}\left\langle\tilde{n}^{2}(z)\right\rangle\right] d z,
$$

so that by defining a length scale:

$$
l_{z}(z)=\frac{\iint S\left(k_{x}, k_{y}, 0 ; z\right) d k_{x} d k_{y}}{\left\langle\tilde{n}^{2}(z)\right\rangle},
$$

then the result can be stated in conventional form $[8,9]$ :

$$
\left\langle\tilde{N}^{2}\right\rangle=\int\left\langle\tilde{n}^{2}(z)\right\rangle l_{z}(z) d z
$$

In this manner, the selection rule $k_{z}=0$ is taken into account by this parameter $l_{z}$, simply representing the ratio of the fluctuation power spectral density with $k_{z}=0$ to the total fluctuation power. We discuss the dependence of $l_{z}$ further in Sec. 4.

Because of finite correlation length, any correlation function must have some non-zero amplitude at $k_{z}=0$. For example, a realistic correlation function might have a functional form $\Gamma(\Delta z)=\exp \left(-\Delta z^{2} / \mathcal{L}^{2}\right) \exp \left(i k_{z 0} \Delta z\right.$ ) (where $\mathcal{L}$ is the coherence length and $k_{z 0}$ is the peak wave-number in the $z$ direction), so that $l_{z}=\mathcal{L} \exp \left(-\mathcal{L}^{2} k_{z 0}^{2} / 4\right) /(2 \sqrt{\pi})$. This shows that if $\mathcal{L}=\lambda, l_{z}$ is close to $\mathcal{L}$, however for longer correlation lengths, $l_{z}$ rapidly becomes smaller (coherent structures tend to cancel each other out). From this it is seen that a wave-packet with non-zero peak $k_{z 0}$ will produce a line-integrated signal through its finite correlation length.

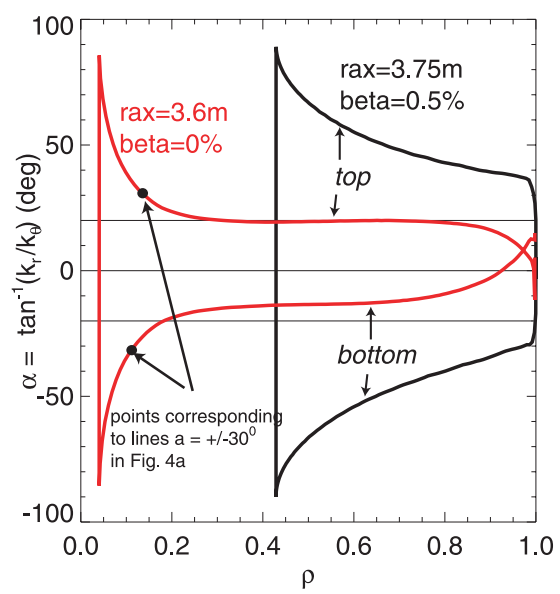

Fig. 3 The angle of the line corresponding to the constraint $k_{z}=$ 0 , as a function of $\rho$ for different magnetic configurations.

\section{Forward Modelling of Signal from Spectrum in Flux Coordinates}

Though we have shown that components with $k_{z} \neq 0$ are not detected, we now concentrate on the forward problem, i.e. modelling of the expected signal from a given spectrum in flux coordinates. In order to relate the measurements to the fluctuation spectrum expressed in terms of radial and poloidal components, we define the following local coordinate system: $\boldsymbol{r}=\nabla \rho$ (where $\rho$ is the flux surface coordinate), $\boldsymbol{B}$ (magnetic field vector), and a poloidal direction vector $\hat{\boldsymbol{\theta}}=\hat{\boldsymbol{B}} \times \hat{\boldsymbol{r}}$. Given $\hat{\boldsymbol{B}}$ is perpendicular to the projected fluctuation, we are interested in the projection in the $\hat{z}$ direction, as well as in the direction perpendicular to the field $\hat{\boldsymbol{p}}=\boldsymbol{B} \times \hat{z}$. Thus, the coordinate transformation modifies the wave-vectors via:

$$
\left(\begin{array}{l}
k_{z} \\
k_{p}
\end{array}\right)=\left(\begin{array}{ll}
\hat{\theta}_{z} & \hat{r}_{z} \\
\hat{\theta}_{p} & \hat{r}_{p}
\end{array}\right)\left(\begin{array}{c}
k_{\theta} \\
k_{r}
\end{array}\right),
$$

where the components of the matrix are computed from the flux surface geometry, and can be shown to have the structure of a simple rotation matrix (with unity Jacobian), since $\hat{\theta}_{p}=-\hat{r}_{z}$ and $\hat{\theta}_{z}=\hat{r}_{p}$. Expanding the first row of Eq. (9), the condition for detection upon line-integration, $k_{z}=0$ implies that the detected fluctuation components lie on a line in $\left(k_{r}, k_{\theta}\right)$ space with angle $\alpha$ such that $\tan \alpha=$ $-\hat{\theta}_{z} / \hat{r}_{z}$.

Calculations of the angle $\alpha$, as a function of $\rho$ (having mapped $z$ to $\rho$ ) for different magnetic axis positions $\left(R_{\mathrm{ax}}\right)$ is given in Fig. 3. For the configuration with $R_{\mathrm{ax}}=3.6 \mathrm{~m}$, the sight line passes very close to the core (as in Fig. 1), so that $\alpha$ should be close to zero for most of the viewing line. However, as indicated, $\alpha \sim 20^{\circ}$, because $\hat{\boldsymbol{\theta}}$ has a significant vertical component, and $\hat{\boldsymbol{r}}$ has a significant toroidal component (since the sight line is slightly semi-tangential with an angle of $\sim 6^{\circ}$ to vertical). For $R_{\mathrm{ax}}=3.75 \mathrm{~m}$, the angle $\alpha$ is larger, being around $45^{\circ}$ at the edge of the plasma.

For the sake of example, we prescribe the local spec- 
trum of turbulence in flux coordinates, $S_{\mathrm{flx}}\left(k_{r}, k_{\theta} ; z\right)$, whose $\left(k_{r}, k_{\theta}\right)$ dependence is plotted in Fig. 4 (a). The distribution along $z$ is assumed to be centred at $z_{0}$ with width as narrow as $l_{\text {turb }}$ (several correlation lengths), so that it projects with a unique angle $\chi_{p}\left(z_{0}\right)$ into the $\left(k_{x}, k_{y}\right)$ plane. For this example, we have considered fluctuations propagating in the electron diamagnetic poloidal direction, and both inwards and outwards in the radial direction, with some radial asymmetry, in order to show the effect this can have on the detected signal. The spectral density function is transformed from flux coordinates to beam aligned coordinates (along and perpendicular to it) via the $1: 1$ transform $S_{\mathrm{p}}\left(k_{p}, k_{z} ; z\right)=S_{\mathrm{flx}}\left(k_{r}, k_{\theta} ; z\right)$. This transformation is depicted in Fig. 4 (a) as a rotation of the coordinate axis $\left(k_{r}, k_{\theta}\right) \rightarrow\left(k_{p}, k_{z}\right)$ through angle $\alpha$. We consider the transformed signal for two possible values of $\alpha$. The slice $S_{p}\left(k_{p}, 0\right)$, which contributes to the line-integral signal, is plotted for each value of $\alpha$ in Fig. 4 (b). Since only components along the line $k_{r} / k_{\theta}=\tan \alpha$ (such that $k_{z}=0$ ) can contribute to the line-integral, the contribution to $\left\langle\tilde{N}^{2}\right\rangle$ will generally depend strongly on $\alpha$ (determined only by the position $z$ along the sight line and flux surface geometry), even for constant local $\left\langle\tilde{n}^{2}\right\rangle$ because $S_{\text {flx }}$ is anisotropic. This is equivalent to there being a dependence $l_{z}(\alpha)$.

It is clear that $\alpha$ is asymmetric with respect to fluctuation components measured on the top and on the bottom. This implies that any asymmetry in $k_{r}$ of $S_{\mathrm{flx}}\left(k_{r}, k_{\theta} ; z\right)$ may result in a different line integrated fluctuation profile on the top (for $z=z_{t}$ corresponding to $\rho=\rho_{0}$ ), compared with the bottom (with $z=z_{b}$, having the same $\rho=\rho_{0}$ ), even if $S_{\mathrm{flx}}\left(k_{r}, k_{\theta} ; z_{t}\right)=S_{\mathrm{flx}}\left(k_{r}, k_{\theta} ; z_{b}\right)$, i.e. fluctuation properties are constant on a flux surface. This is illustrated by comparing the fluctuation profiles $S_{p}\left(k_{p}, 0 ; z_{t}\right.$ ) (for which $\alpha=30^{\circ}$ ) and $S_{p}\left(k_{p}, 0 ; z_{b}\right)$ (for which $\alpha=-30^{\circ}$ ) in Fig. 4 (b). The radially asymmetric spectrum produces an apparent up/down asymmetry in the deduced local fluctuation intensities. It is possible that the radial asymmetry may also relate to the radial flux. Such kinds of asymmetric fluctuation amplitude profiles are regularly observed in the measurements [5, 11] (and see Fig. 2).

Finally, the spectrum can be transformed from $S_{p}\left(k_{p}, 0 ; z\right)$ to cartesian coordinates $S\left(k_{x}, k_{y}, 0 ; z\right)$ by the relations $k_{x}=k_{p} \cos \chi_{p}(z), k_{y}=k_{p} \sin \chi_{p}(z)$, and based on Eq. (5), the line-integrated spectrum in the image plane can thus be related to $S_{\mathrm{p}}$ through:

$$
\bar{S}\left[-k_{p} \sin \chi_{p}(z), k_{p} \cos \chi_{p}(z)\right]=S_{\mathrm{p}}\left(k_{p}, 0 ; z\right) \frac{d z}{d \chi_{p}(z)} .
$$

For the above analysis, we shall give a short mention about the role of Doppler shifting by poloidal flows. The frequency dependent flux surface function $S_{\text {flx }}\left(k_{r}, k_{\theta}, \omega ; z\right)$ would be transformed by a poloidal rotation $v_{\theta}$ to $S_{\mathrm{flx}}\left(k_{r}, k_{\theta}, \omega+v_{\theta} k_{\theta} ; z\right)$. Considering that the frequency of turbulence in the plasma frame is normally considerably less than the Doppler shift [11], lower frequency (a) model function $\mathrm{S}_{\mathrm{ffx}}\left(\mathrm{k}_{\mathrm{r}}, \mathrm{k}_{\theta}\right)$

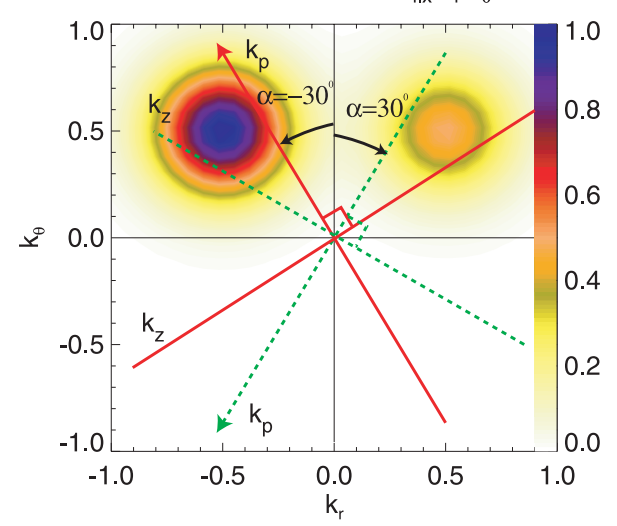

(b) Slices along line $\mathrm{k}_{\mathrm{p}}$ with $\mathrm{k}_{\mathrm{z}}=0$ for different $\alpha$

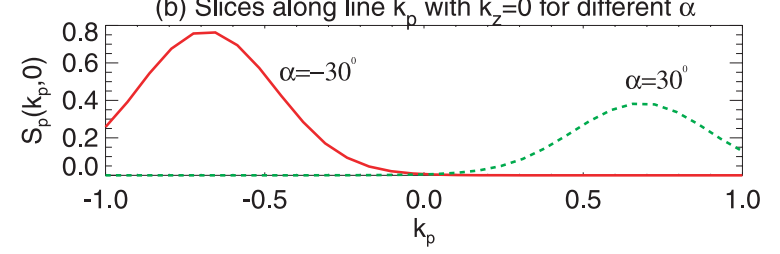

Fig. 4 (a) Example spectrum in $\left(k_{r}, k_{\theta}\right)$ space, together with the axis projections for $\alpha= \pm 30^{\circ}$. (b) Projected spectrum along the line of sight such that $k_{z}=0$, for the same values of $\alpha$ as indicated in (a).

components will intrinsically contain only radial components (with small $k_{\theta}$ ), while the higher frequency components will have some poloidal contribution. Therefore, the frequency of components towards the core of the plasma, where $\alpha \sim 90^{\circ}$, should be low, while components at the edge should have a high peak frequency.

When the sightline is tangent to the flux surface ( $\alpha=90^{\circ}$ ), the system is sensitive to fluctuations with $k_{\theta}=0$. However, in this small $k$ limit, the transformation of $S_{\mathrm{flx}}\left(k_{r}, k_{\theta} ; z\right) \rightarrow S_{\mathrm{p}}\left(k_{p}, k_{z} ; z\right)$ breaks down because the curvature radius of the flux surface is comparable with the poloidal wavelength; in this case the correlation function would have to be projected to determine line integration effect, $\Gamma_{\mathrm{flx}}(\Delta \rho, \Delta \theta ; z) \rightarrow \Gamma_{\mathrm{p}}(\Delta p, \Delta z ; z)$. However, it is clear that the signal should be sensitive to components with small $k_{\theta}$. This includes features such as GAMS and low $m$ MHD modes. Though such detectable fluctuations would have $k_{\theta} \approx 0$, the $k$ transfer function of the PCI system only admits components with $k_{p}=k_{r} \gtrsim 1 \mathrm{~cm}^{-1}$, implying that the detected fluctuations at this point must have fine radial structure. This is compatible with the characteristics of GAMS (long poloidal wavelength, short radial wavelength). The same conclusion about the detection of zonal flows with PCI was also stated in [12]. Though zonal flows may produce no net density fluctuation (completely in the potential field), the associated GAM may have a density fluctuation component; one experimental study has shown that for the GAM, $\tilde{n} / n \sim 0.1 \tilde{\phi} / \phi$ (where $\phi$ is the plasma potential) [13]. 


\section{Discussion}

We have shown that a line-integrated signal is sensitive only to the Fourier component of fluctuations with $k_{z}=0$. By defining a length scale in Eq. (7), the rms line-integrated fluctuation can be related to the rms of the local fluctuation. If the spectrum in flux coordinates, $S_{\mathrm{flx}}\left(k_{r}, k_{\theta} ; z\right)$ were isotropic perpendicular to the field lines, then $\left\langle\tilde{n}^{2}\right\rangle$ could be reconstructed unambiguously from Abel inversion of the measured $S_{\mathrm{p}}\left(k_{p}, 0 ; z\right)$. However, in general, no such assumption can be made. For example, measurements from beam emission spectroscopy in DIII-D of the local power spectrum shows a non-zero poloidal peak wave-number, and a zero peak radial wave-number [14]. Therefore we must accept that line-integral signals cannot fundamentally deliver the local fluctuation power without some assumptions or further information, for example, probing the same volume from a different direction, or probing the same flux surface at different points with different angle $\alpha$. This is achieved with 2D PCI for 2 points, since $\alpha(-\rho) \approx-\alpha(\rho)$, as shown in Fig. 3. However, 2 values of $\alpha$ for every $\rho$ may not be enough to calculate $\left\langle\tilde{n}^{2}\right\rangle$ accurately. For practical use, we can generally make the assumption that $l_{z} \sim \lambda / 4$, where $\lambda$ is peak the wavelength. This relation was shown to hold on the TCA Tokamak [8] for homogeneous turbulence as $l_{z}$ and $\lambda$ increase with temporal frequency. Further consideration of these issues is the topic of future work.

\section{Acknowledgements}

The authors would like to thank J.C. Rost of MIT, D. Mikkelson of PPPL and Prof. K. Itoh of NIFS for valuable discussions, the reviewer for insightful comments and the LHD experimental group for machine operation. This work was supported by a grant-in-aid from the Japan Society for the Promotion of Science (JSPS) and the National Institute for Fusion Science budget NIFS05ULHH511.

[1] H. Wiesen Plas. Phys. Control. Fus. 28(8), 1147 (1986).

[2] L. Vyacheslavov et al., In these proceedings.

[3] A.L. Sanin et al., Rev. Sci. Instrum. 75(10), 3439 (2004).

[4] L.N. Vyacheslavov et al., IEEE trans. plas. sci. 33, 464 (2005).

[5] C.A. Michael et al., Rev. Sci. Instrum. (10), 10E923 (2006).

[6] M. Porkolab et al., IEEE Trans. Plas. Sci. 34(2), 229 (2006).

[7] A. Marinoni et al., Rev. Sci. Instrum. 77, $10 \mathrm{E} 929$ (2006).

[8] H. Wiesen et al., Plas. Phys. Control. Fus. 30(3), 293 (1988).

[9] S. Coda. PhD thesis, MIT, 1997.

[10] W.H. Carter and E. Wolf Opt. Commun. 67(2), 85 (1988).

[11] C.A. Michael et al., Proc. EPS, Rome P4.114. (2006).

[12] S. Coda et al., Phys. Rev. Lett. 86(21), 4835 (2001).

[13] A.V. Melnikov et al., Plas. Phys. Control. Fus. 48(4), S87 (2006).

[14] G.R. McKee et al., Rev. Sci. Instrum. 74(3), 2014 (2003). 